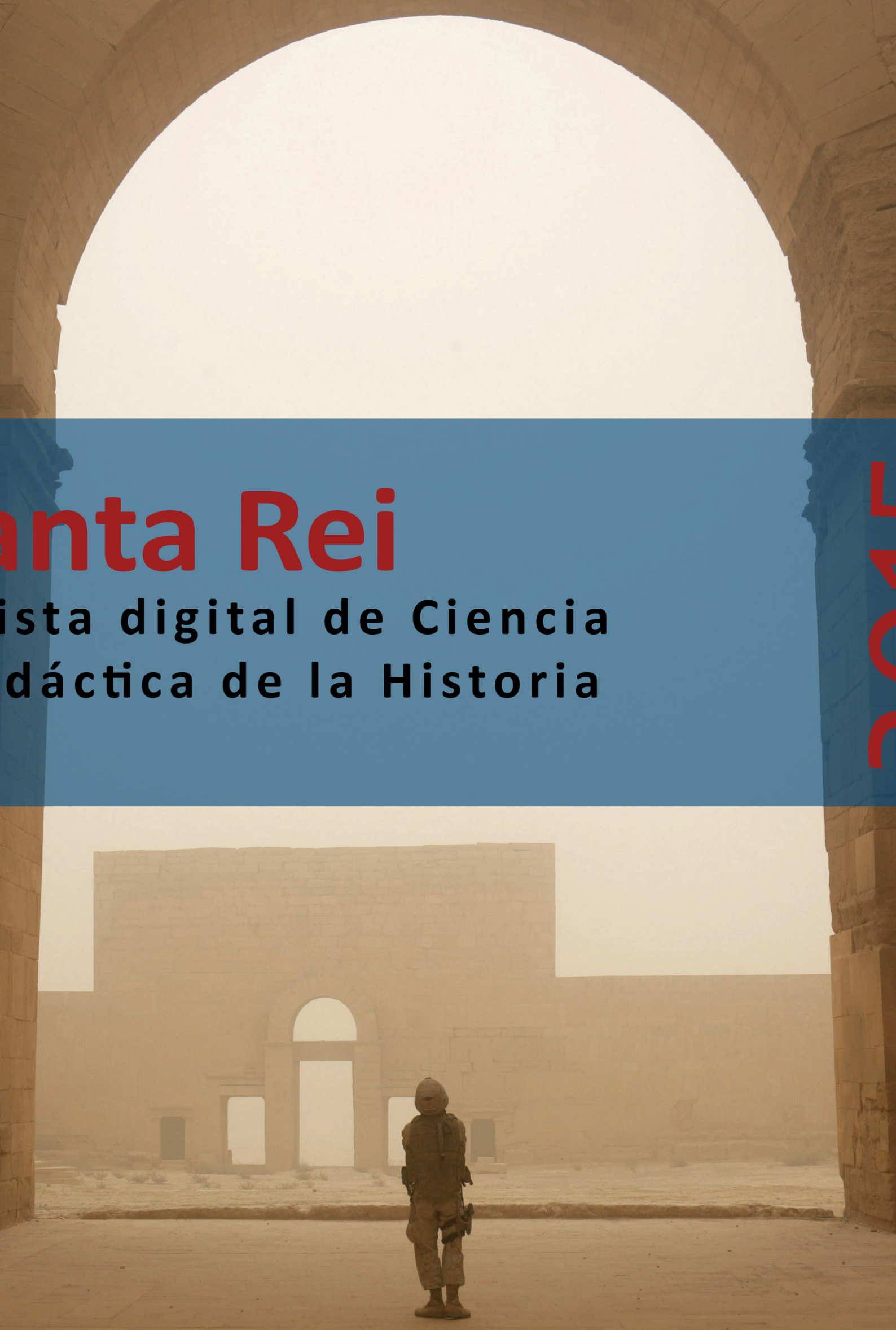

Revista digital de Ciencia y Didáctica de la Historia 


\section{Panta Rei \\ Revista Digital de Ciencia \\ y Didáctica de la Historia}

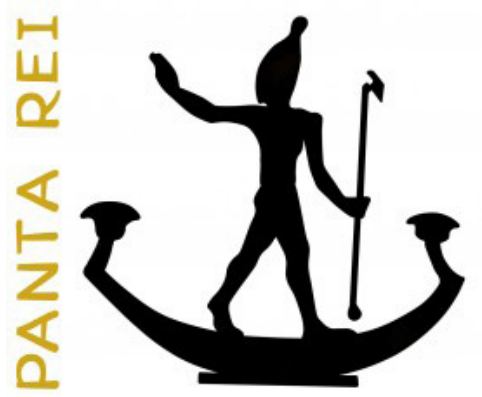

\section{5}

Revista anual

Fecha de inicio: 1995

Revista Panta Rei. pantarei@um.es

Edita:

Centro de Estudios del Próximo Oriente y la Antigüedad Tardía - CEPOAT

Edificio Universitario Saavedra Fajardo.

Universidad de Murcia

C/ Actor Isidoro Máiquez, 9

30007 - MURCIA - ESPAÑA

Teléfono: (+34) 868883890

cepoat@um.es

Web: www.um.es/cepoat/pantarei

Edición 2015

ISSNe: 2386-8864

ISSN: 1136-2464

Depósito legal: MU-966-1995
cepoAt

UNIVERSIDAD DE MURCIA centro de estudios del próximo oriente y la antigüedad tardía

En Portada: Ruinas de Hatra (Irak) (Fotografía de: Lance Cpl. Albert F. Hunt, U. S. Marine Corps).

Responsables de los textos: Sus autores.

Responsable de la presente edición: Consejo Editorial Panta Rei. 


\section{CONSEJO DE REDACCIÓN}

\section{Coordinador editorial}

Egea Vivancos, Alejandro

[Didáctica de las Ciencias Sociales, UMU]

\section{Editores}

Botí Hernández, Juan Jesús

[CEPOAT, UMU]

Meseguer Gil, Antonio José

[CEPOAT, UMU]

Sáez Giménez, David Omar

[CEPOAT, UMU]

Sánchez Mondéjar, Celso Miguel

[CEPOAT, UMU]

\section{Secretaria}

Arias Ferrer, Laura

[Didáctica de las Ciencias Sociales, UMU]

\section{Responsable informático}

Martínez García, José Javier

[CEPOAT, UMU]

\section{Traducción y corrección lingüística}

Martínez Martínez, Cristina

[Sociedad Española de Lenguas Modernas]

\section{CONSEJO ASESOR}

Albero Muñoz, M. ${ }^{a}$ del Mar [H. ${ }^{a}$ del Arte, UMU]

Chapman, Arthur

[History Education, UCL, Reino Unido]

Cobacho López, Ángel

[Derecho, UMU]

Egea Bruno, Pedro M. ${ }^{\text {a }}$

[Historia Contemporánea, UMU]

García Atienzar, Gabriel

[Prehistoria, UA]

González Monfort, Neus

[Didáctica de las Ciencias Sociales, UAB]

Haber Uriarte, María

[Prehistoria, UMU]

Irigoyen López, Antonio

[Historia Moderna, UMU]

Mahony, Simon

[Digital Humanities, UCL, Reino Unido]

Marsilla de Pascual, Francisco Reyes

[Técnicas historiográficas, UMU]

Miralles Maldonado, José Carlos

[Filología Clásica, UMU]

Molina Gómez, José Antonio

[Historia Antigua, UMU]

Noguera Celdrán, José Miguel

[Arqueología, UMU]

Pérez Molina, Miguel Emilio

[Filología Clásica, UMU]

Prados Martínez, Fernando

[Arqueología, UA]

Sánchez Ibáñez, Raquel

[Didáctica de las Ciencias Sociales, UMU]

Sancho Gómez, Miguel Pablo

[Educación, UCAM]

Vilar García, María José

[Historia Contemporánea, UMU] 

Artículos

El patrimonio arqueológico iraquí y su destrucción a lo largo del tiempo.

Fernando Espejel Arroyo.

Tacita Muta y el silencio femenino como arma del patriarcado romano.

Sara Casamayor Mancisidor.

Espartaco en la arqueología.

Juan Luis Posadas.

La batalla de Andagoste.

Luis Amela Valverde.

Las trabas para una formación arqueológica inclusiva.

Ana Samaniego Espinosa.

Repensando las narrativas nacionales: Un análisis del origen, transmisión e influencia en el aprendizaje histórico.

César López Rodríguez

El profesor como predicador, provocador y ermitaño. Un pre-texto para pensar las tareas de la didáctica de la historia.

Enrique Ibáñez Rojo

Entrevista

Evaluando el desarrollo de la competencia histórica en la enseñanza de la Historia en España. Entrevista a Jesús Domínguez Castillo.

María José Morales Rodríguez.

Reseñas

II Congreso de Jóvenes Investigadores del Mundo Antiguo de la Universidad de Murcia.

Pedro David Conesa Navarro y Juana M. ${ }^{a}$ Martínez Molina.

II Jornadas Internacionales de Didáctica de la Historia.

Eusebio Manuel Vidal Abellán.

Dölger, F. J. (2015). La luz de Cristo. Madrid: Ediciones Encuentro. 98 págs.

Alejandro Ruiz Sánchez.

Cooper, H. (ed.) (2013). Teaching History Creatively. Londres: Routledge. 185 págs.

Marta Gil de Sola Bellas.

El arte paleolítico en El Carnicero de Chabrol.

Alberto Lombo Montañés.

Normas de publicación/Publishing rules 



\title{
Espartaco en la arqueología
}

\author{
Spartacus in Archaeology
}

Juan Luis Posadas ${ }^{1}$

Universidad Nebrija

Recibido: $29 / 04 / 15$

Aceptado: 15/07/15

Para citar este artículo: Posadas, J. L. (2015). Espartaco en la arqueología. Panta Rei. Revista Digital de Ciencia y Didáctica de la Historia, 43-49.

ISSNe: 2386-8864

DOI: http://dx.doi.org/10.6018/pantarei/2015/3

\section{Resumen}

Desde 2011 estamos desarrollando una línea de investigación sobre las fuentes escritas para la rebelión de Espartaco. En este artículo vamos a repasar las pocas evidencias arqueológicas de dicha rebelión, sobre todo la pintura aparecida hace tiempo en Pompeya donde se representa a un jinete con una inscripción en osco referida a un Spartaks. La hipótesis es que dicha representación alude a un episodio concreto de dicha rebelión fechable en el año 73 a. C. (la derrota y muerte del pretor Cosinio a manos de Espartaco).

\section{Palabras clave}

Historia Antigua, Arqueología, Historia de Roma, Epigrafía

\section{Abstract}

Since 2011 we are developing a research about the written sources on the rebellion of Spartacus. In this paper we are to review some archaeological evidences on that rebellion. The most important of these is the wall graffito discovered some decades ago in Pompey representing a horseman with an inscription in Oscan language referring to a Spartaks. Our hypothesis is that this painting represents a specific episode of this rebellion dated in $73 \mathrm{~b}$. C. (the defeat and murder of Praetor Cosinius by Spartacus).

\section{Key Words}

Ancient History, Archaeology, Roman History, Epigraphy

1 Para contactar con el autor: Juan Luis Posadas. Profesor. Dpto. de Lenguas Aplicadas y Educación. Universidad Antonio de Nebrija. Campus Dehesa de la Villa. C/ Pirineos 55, 28040 Madrid.

jposadas@nebrija.es 


\section{Introducción}

A diferencia de las fuentes escritas, hay poquísimas huellas materiales que se puedan asignar a la rebelión de Espartaco. Es muy sorprendente este hecho, habida cuenta la amplitud de la rebelión, el itinerario seguido por los rebeldes, y las supuestas obras de ingeniería acometidas por los romanos para contenerla (como el muro en la "punta" de la bota italiana). Es de resaltar que, a pesar de que se haya investigado in situ en la zona de las crestas de Melia, no se ha encontrado ninguna evidencia por ahora de dicho muro. Gabba (1958) cree que el muro se situó entre Pizzo y Soverato. Strauss (2001) lo sitúa en las crestas del Melìa, entre Maratea y Roseto Capo Spulico. Nosotros creemos que dicho muro debió de tratarse de una simple zanja con defensas en madera, que difícilmente puedan haber dejado más que débiles rastros en el terreno (Posadas, 2012). Sin una prospección sistemática de la zona será difícil probar una u otra localización.

En el caso de las guerras serviles de Sicilia, acaecidas unos treinta años antes de la rebelión de Espartaco, hay más fuentes arqueológicas, como las monedas emitidas por los autoproclamados reyes rebeldes o algunas balas de proyectil romano con inscripciones alusivas a dichas guerras serviles o a los generales que comandaron los ejércitos implicados en su aplastamiento².

Es importante analizar algunas supuestas evidencias arqueológicas sobre la rebelión para ver si aportan datos al estudio de la misma o si nos pueden servir como guía para fijar el itinerario de los ejércitos rebeldes y/o romanos.

\section{Algunos hallazgos arqueológicos}

Como ya hemos señalado, no hay fuentes arqueológicas claras sobre la rebelión de Espartaco. Las más importantes serían los campamentos de los diferentes ejércitos, la localización de alguna de las batallas que se libraron en aquella rebelión, o el estudio in situ del muro construido para contener a Espartaco en el sur de la bota italiana. También sería muy importante localizar balas de proyectil o inscripciones alusivas a esta rebelión. Por ejemplo, los edictos de los pretores convocando las reclutas ad tumultum (Posadas, 2015), o algún texto relativo a la devolución de los esclavos prisioneros a sus legítimos dueños, inscripciones que tienen precedentes en Sicilia tras las guerras serviles.

En el caso de la rebelión de Espartaco, solo podemos citar, y de manera hipotética, dos datos arqueológicos. El primero sería la destrucción del templo de Apolo Aleo de la localidad calabresa de Crimissa (cerca de Cirò Marina), asignada por Orsi (1932) en una primera fase a la guerra púnica en relación con el ejército de Aníbal, y en una segunda fase y definitiva, a la guerra de Espartaco.

La segunda fuente arqueológica es muy interesante porque nos da cuenta del itinerario seguido por los rebeldes en el año 72 a. C. Se trata de un tesorillo enterrado en la localidad de Policoro, en la Basilicata. Policoro está situada a unos pocos kilómetros de la ciudad griega de Heraclea. Según su excavador (Siciliano, 1974-75), este tesorillo estaba compuesto de más de quinientas monedas y él lo dató en el año 72 a. C. (año de la última emisión de las mismas).

Al parecer, siempre según el arqueólogo mencionado, el tesorillo fue enterrado con evidente urgencia por algún rico propietario. Es posible proponer que ello fue debido a la inminente llegada de bandidos o quizá de los propios rebeldes de Espartaco. Ello porque sabemos que en ese año llegaron a la región (Posadas, 2012). Es de señalar que cerca de Policoro se encontraba la localidad de Thurii (Turios), que en nuestra opinión llegó a ser una especie de capital de Espartaco (Posadas, 2012).

2 Shaw (2001) menciona la bala de proyectil con la inscripción "Lucio Pisón, hijo de Lucio, cónsul" (CIL I, 847), la moneda que alude a la victoria de Manio Aquilio en la segunda guerra servil, y otras muchas balas de proyectil con textos referentes a las divinidades de los esclavos en dicha guerra. 


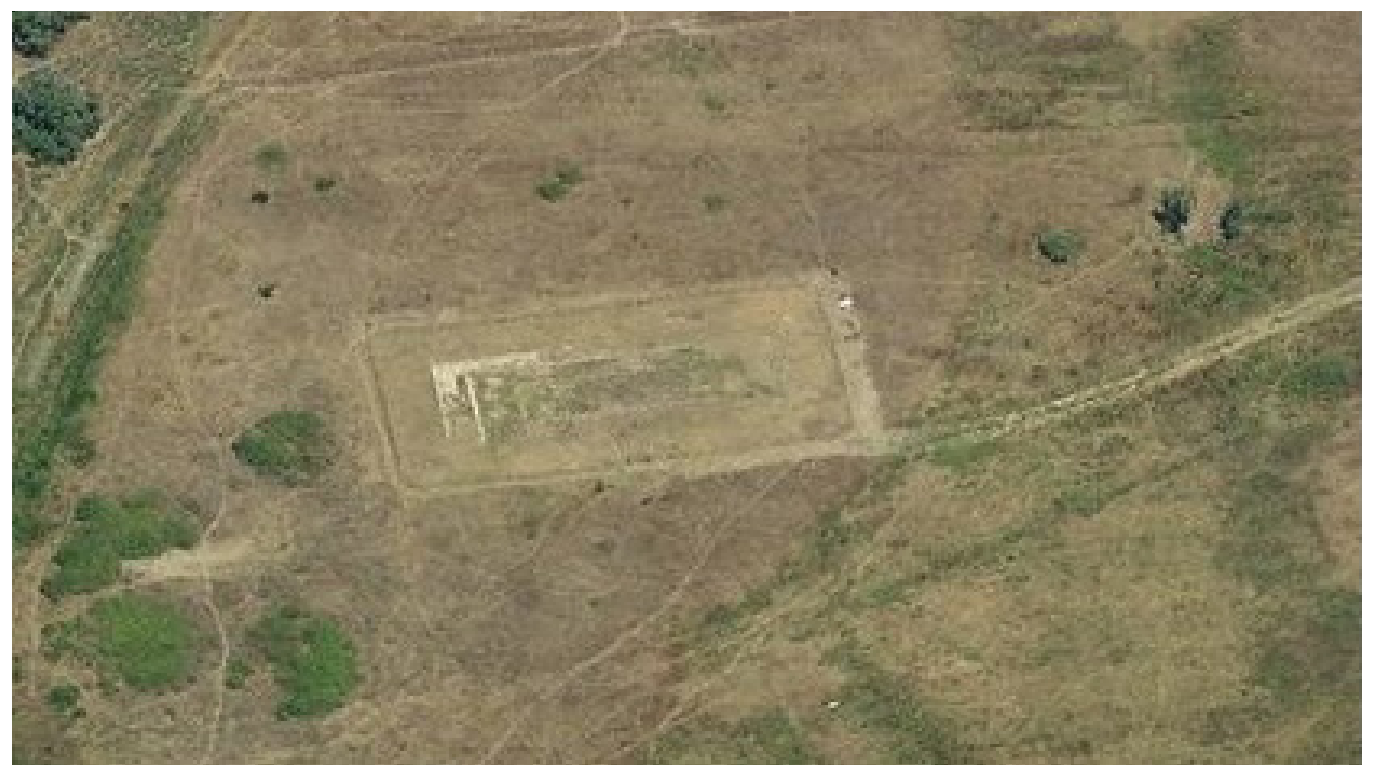

Figura 1: Templo de Apolo. Posible localización del templo de Apolo en Crimissa. Alrededor del templo se situarían los restos, aún sin excavar, de la localidad, que posiblemente también fuera afectada por el saqueo de los rebeldes de Espartaco.

\section{La pintura de Pompeya}

Desde nuestro punto de vista, la fuente iconográfica más importante sobre la rebelión de Espartaco que nos ha legado la arqueología es la pintura parietal encontrada en la pared de una casa de Pompeya. En concreto es la hallada en la casa atribuida a M. Fabio Amando (Maiuri, 1939), catalogada como "casa del sacerdos Amandus" (I, 7, 7). Esta pintura se encontraba en muy mal estado. Además, se hallaba bajo varias capas de pintura añadidas posteriormente. Para colmo de males, fue descubierta en los años treinta del siglo pasado, por lo que las técnicas deficientes utilizadas tanto para retirar las capas de pintura posteriores como para documentar el descubrimiento y preservarlo, han terminado por destruir casi irremediablemente el grafito.

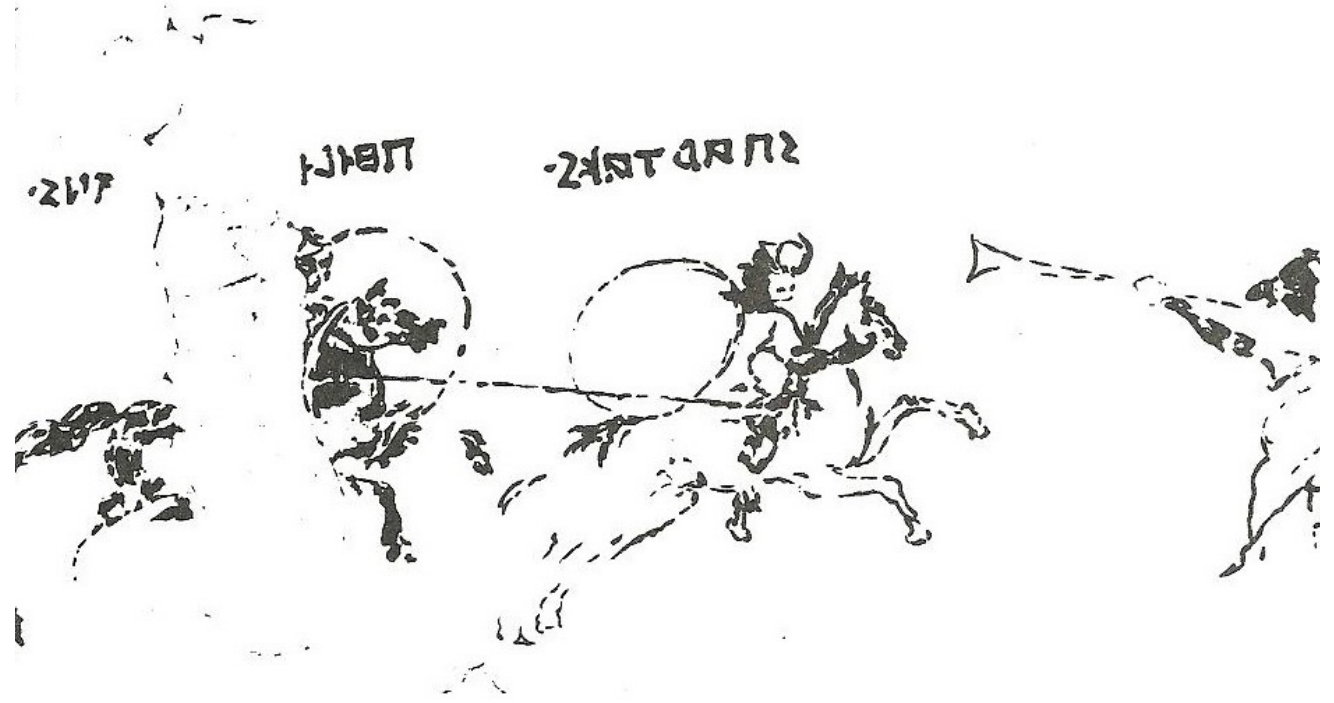

Figura 2: Grafito encontrado en la entrada de la casa del sacerdos Amandus (I, 7, 7) (Maiuri, 1939, p. 5). 
En la pintura parietal se veía a dos hombres a caballo con escudo redondo grande y pesado, persiguiendo uno -blandiendo una lanza- a otro. Frente al "perseguido", y de cara a él, aparecía un personaje con una tuba o trompa y con una indumentaria de piel de animal. Este personaje se ha interpretado como un músico romano que daría la alerta ante la llegada de perseguidor y perseguido al campamento. También aparecen, algo distanciados, dos hombres luchando que podrían ser gladiadores o soldados, e incluso podrían pertenecer a una pintura distinta.

La pintura presenta, además, dos textos en lengua osca, la hablada en la zona de Pompeya al menos durante la época republicana. La figura ecuestre central presenta encima de ella el texto (escrito de derecha a izquierda) SPARTAKS ("Espartaco" en osco). La segunda figura presenta un texto encima con las letras PHILI(CS) ... NS ("afortunado ...ns").

Las circunstancias de dicho hallazgo son:

- Un grafito aparecido en Pompeya (año 79 d. C.), pero tras varias capas de pintura que podrían hacer retroceder su datación a unos 150 años antes (hacia el año 70 a. C.).

- Representación de un jinete que persigue a otro que se dirige hacia un músico que parece romano.

- Una inscripción en lengua osca datable en la primera mitad del siglo I a. C.

- Dicha inscripción puede traducirse como "Espartaco afortunado [...]ns".

A simple vista de tales datos y circunstancias, la interpretación lógica de dicho grafito es que representa al líder de la rebelión de los gladiadores de Capua: misma fecha, temática militar, lengua de la región desaparecida a mediados del siglo en que ocurrió la rebelión, y, sobre todo, nombre de Espartaco.

A estos hechos, habría que añadir, además, que el escenario de los primeros actos de la rebelión de Espartaco (el Ludus de Capua, la huida al Vesubio, las derrotas romanas de los ejércitos pretorianos, etc.) es muy próximo en el espacio al lugar donde apareció este grafito, Pompeya. La procedencia social del pintor, baja por ser el grafito un arte eminentemente popular, y además en lengua osca, la lengua probablemente hablada por la capa más humilde de la ciudad, hace que podamos inferir una cierta simpatía del pintor por Espartaco, el líder de los oprimidos de la región. Esta identificación del jinete del grafito con el Espartaco de la rebelión ha sido apoyada por algunos autores, entre ellos Shaw (2001) y Fields (2009).

Pero Beard no cree en dicha identificación y piensa que se trata de una representación de "algún tipo de combate de gladiadores" (Beard, 2009, p. 67). Dada la eminencia de la autora, conviene comentar algo al respecto: por supuesto, la lucha de gladiadores a caballo existía en esta época. Había al menos dos tipos de gladiadores a caballo: los andabatae y los equites (Grant, 1995). Según esto, es posible que el Espartaco del grafito realmente fuera un gladiador andabata a caballo. También hay paralelos en Pompeya de combates gladiatorios donde aparecen músicos (CIL IV, 1071). Sin embargo, los músicos de dichos graffiti parecen flautistas, no trompetistas. Y, desde luego, no visten con pieles de animales. Desde mi punto de vista, el argumento de Beard es valioso pero no concluyente.

Otro experto conocedor de la arqueología pompeyana, Pesando, concluye que la pintura se refiere a un combate gladiatorio ritual que, debido a la presencia de ciertos elementos arquitectónicos, como un altar, habría tenido lugar en el foro de Pompeya (Pesando y Guidobaldi, 2006). Si bien el autor puede tener razón en ver un ambiente ritual en la representación, no explica la mención del nombre de Espartaco, por lo que no tendría sentido que dicha escena hubiera ocurrido en el foro pompeyano; a no ser que Pesando piense que Espartaco participó en unos juegos gladiatorios en dicha ciudad. Lo que parece claro y contundente es que la representación alude no tanto a un combate gladiatorio sino a un combate a caballo en el que uno persigue y otro huye.

El experto que ha estudiado este grafito con mayor profundidad, en la medida de nuestros conocimientos, Kolendo, ha convenido en su estudio lo siguiente: el tal Spartaks (nombre tracio) era tracio, sí, y gladiador, también, pero desconocido. Según Kolendo, no puede ser el gladiador tracio Espartaco líder de la rebelión (Kolendo, 1980).

Siguiendo el argumento de la navaja de Ockham, que la explicación más sencilla es la más 
probable, aunque no necesariamente la verdadera, parece lógico suponer que una pintura de la primera mitad del siglo I a. C. sobre un gladiador tracio de nombre Espartaco, localizada en la región donde se desarrolló una rebelión, se refiere al Espartaco líder de los rebeldes (Posadas, 2012). siguiente:

Desde nuestro punto de vista, la interpretación correcta del texto y de la pintura podría ser la

Sobre el texto, este dice: Spartaks phili [...]ns. ¿Podría reconstruirse el texto como Spartaks phili[cs Cosi]ns? ("Espartaco - afortunado Cosinio").

Otra cuestión es quién en el grafito es Espartaco y quién sería [Cosi]ns. Nuestra interpretación es que, al ser el osco un idioma que se lee al revés que el alfabeto latino, de derecha a izquierda, pero al ser el "sentido de la marcha" de los jinetes de izquierda a derecha, los rótulos con los nombres Spartaks y [Cosi]ns no deben aplicarse a los personajes que están inmediatamente debajo, sino al revés: Spartaks sería el jinete "perseguidor" y [Cosi]ns el perseguido.

Según nuestra hipótesis, y según la lectura que proponemos, la pintura pompeyana de la casa de Fabio Amandio, podría representar un episodio de la guerra de Espartaco que viene bien explicado en el relato de Plutarco, probablemente tomado de un texto desaparecido de las Historias de Salustio ${ }^{3}$ :

Después, cuando Cosinio fue enviado con un gran ejército como consejero y colega de Publio, Espartaco estuvo espiándolo y, mientras Cosinio se estaba bañando cerca de Salinas, estuvo a punto de capturarlo. Cosinio logró escapar a duras penas y con dificultad; pero Espartaco se apoderó inmediatamente de su bagaje, lo siguió de cerca y lo persiguió; se adueñó de su campamento y mató a muchos de sus hombres. Cosinio también cayó.

$$
\text { (Plu., Crass. 9, 5-6) }
$$

Según este texto, Espartaco persiguió personalmente a Cosinio, quien llegó hasta su campamento, tomándolo el primero y matando al dicho Cosinio. La escena, según nuestra interpretación, representaría la persecución de Espartaco con lanza a Cosinio, quien ha perdido la suya, y que se dirige hacia un músico "romano" (el tocador de tuba con pieles de animales) con que podría representar el campamento en el que Cosinio se refugió.

Este sería el episodio más famoso de la rebelión, pues en tiempos antiguos se consideraba algo heroico el duelo individual entre los generales de dos ejércitos, y más si uno arrebataba las armas al otro o, como se sabe, lo mataba en combate singular ${ }^{4}$. Este acontecimiento, que podría haber ocurrido, sería digno de ser inmortalizado en paredes de ciudades cercanas a los acontecimientos, como Pompeya.

Hay que subrayar que esta "fuente arqueológica" de Pompeya sostiene la versión de Plutarco en el sentido de que Cosinio escapó a la persecución de Espartaco -de ahí el calificativo de afortunado que el grafito le da- pero no menciona para nada la muerte del pretor en el campamento, algo que un osco o samnita de la zona sí hubiera señalado.

Téngase en cuenta, a este respecto, que Salinas, el lugar donde ocurrió la persecución y muerte de Cosinio y su ejército a manos de Espartaco, se encuentra entre Pompeya y Herculano ${ }^{5}$. En Pompeya hay una "Puerta Salina" (Veru Sarinu en osco) porque da al camino que va desde dicha ciudad a Salinas (Etienne, 1996). Que un habitante osco-parlante de Pompeya, ciudad cercana a los acontecimientos, dibujara este episodio y calificara a Cosinio como afortunado por escapar de un aguerrido Espartaco, señala lo importante y lo atractivo de la rebelión para los habitantes menos

3 Ya que Salustio se refiere claramente a dicho episodio en un texto desaparecido, del que, sin embargo, queda una referencia en Hist. 3, 94: "Cosinio se lavaba en la fuente de una villa cercana".

4 Espartaco fue alabado por capturar el caballo de otro general romano anterior, Varinio Glabro (App., $B C$ 1.116).

5 En concreto, Plutarco habla de que se estaba dando un baño cerca de Salinas. 
favorecidos de la zona.

Es preciso señalar, en este sentido, que Pompeya se sumó a la rebelión de samnitas y oscos contra Roma en el año 90 a. C. -la guerra de los aliados- y que fue sometida a asedio y tomada por Cornelio Sila un año después (Beard, 2009). Pompeya sufrió destrucciones y mortandad en dicho episodio. La población de lengua osca fue desplazada del poder económico, político y social en Pompeya, y en ella se estableció una colonia de romanos (Fields, 2009). Es evidente que, a los oscos supervivientes, un rebelde como Espartaco que había puesto en fuga a un romano como Cosinio a pocos kilómetros de la ciudad, y solo dieciséis años después de su derrota frente a Roma, dicho episodio les parecería digno de ser recordado con una pintura parietal.

\section{Conclusión}

Hay pocas, poquísimas evidencias arqueológicas de la rebelión de Espartaco. Y una de las que podrían considerarse como tal ha sido puesta en duda por algunos estudiosos. Nos referimos a la pintura parietal o grafito de la casa de M. Fabio Amando en Pompeya. En nuestra opinión, tras proponer una interpretación gráfica de lo representado basada en los dos jinetes y el músico, y una lectura y traducción del texto que pensamos podría ser "Espartaco afortunado Cosinio", consideramos que dicho grafito se relaciona con el episodio de la rebelión datado en 73 a. C., en el que -según Plutarco (Crass. 9.5-6)- Espartaco sorprendió al pretor Cosinio dándose un baño, y le persiguió a punto de capturarle hasta su campamento, que tomó y donde el propio Cosinio murió.

En nuestra opinión, pues, la pintura parietal de Pompeya no solo representa a Espartaco, el gladiador tracio líder de la rebelión de los años 73-71 a. C., sino a Cosinio, el "afortunado" pretor que escapó de Espartaco, aunque después muriera en su campamento. Dicho grafito apoya la versión de Salustio de la que toma su relato Plutarco, da una idea de las armas de Espartaco (escudo circular y lanza), y es un testimonio de que los samnitas y oscos -pues en dicho lenguaje está escrita la inscripción- estaban en minoría en la ciudad y apoyaban a Espartaco en su lucha contra Roma.

\section{Abreviaturas}

CIL I. Corpus Inscriptionum Latinarum, I. Inscriptiones Latinae antiquissimae ad C. Caesaris mortem. Berlín: Berlín-Brandenburg Academy of Sciencies and Humanities. 1863.

CIL IV. Zangemesiter, C. y Schoene, R. Corpus Inscriptionum Latinarum, IV. Inscriptiones parietariae Pompeianae Herculanenses Stabianae. Berlín: Berlín-Brandenburg Academy of Sciencies and Humanities. 1871.

\section{Bibliografía}

Beard, M. (2009). Pompeya. Historia y leyenda de una ciudad romana. Barcelona: Crítica.

Etienne, R. (1996). La vida cotidiana en Pompeya. Madrid: Temas de Hoy.

Fields, N. (2009). Spartacus and the slave war 73-71 B. C. Nueva York: Osprey.

Gabba, E. (1958). Appiani Bellorum Civilium liber primus. Florencia: La Nuova Italia.

Grant, M. (1995). Gladiators. Nueva York: Barnes \& Noble.

Kolendo, J. (1980). Uno Spartaco sconosciuto nella Pompei osca. Le pitture della casa di Amando. Index: quaderni camerti di studi romanistici, 9, 33-40.

Maiuri, A. (1939). Le pitture della case di M. Fabius Amandio, del Sacerdos Amandus, e di P. Cornelius Tege (Reg. I, Ins. 7). Vol. 3. Roma: Monumenti della Pittura Antica scoperti in Italia.

Orsi, P. (1932). Templum Apollinis Alaei ad Crimisa-Promontorium. Atti del Sodalizio Glottologico Milanese, 7-182.

Pesando, F. y Guidobaldi, M. P. (2006). Pompei, Oplontis, Ercolano, Stabiae. Roma-Bari: Laterza.

Posadas, J. L. (2006). Gayo Salustio Crispo: Fragmentos de las Historias. Madrid: Ediciones 
Clásicas.

Posadas, J. L. (2012). La rebelión de Espartaco. Madrid: Sílex.

Posadas, J. L. (2015). La recluta ad tumultum como respuesta equivocada ante la rebelión de Espartaco en el año 73 a. C. En Bravo, G. y González Salinero, R. (eds.). Poder central y poder local. Dos realidades paralelas en la órbita política romana (pp. 123-134). Madrid: Signifer.

Shaw, B. D. (2001). Spartacus and the slave wars. A brief history with documents. Boston-Nueva York: Bedford St. Martin's.

Siciliano, A. (1974-1975). Ripostiglio di monete repubblicane da Policoro. Annali dell'Istituto Italiano di Numismatica, 21-22, 103-154.

Strauss, B. (2010). La guerra de Espartaco. Barcelona: Edhasa.

\section{Fuentes}

Apiano. Guerras Civiles. Libros I-II. Madrid: Gredos. 1985. [Traducción de A. Sánchez Royo]. Plutarco. Vida de Craso. Madrid: Gredos. 2007. [Edición de A. Ledesma].

Salustio. Historias. Madrid: Ed. Clásicas. 2006. [Edición y traducción de J. L. Posadas] 



\section{Panta Rei}

PANTA REI es una revista digital de investigación orientada a la Historia y otras ciencias afines. Su principal objetivo es la transmisión del conocimiento científico, dando una oportunidad también a los jóvenes investigadores que quieren abrirse camino en el estudio de las ciencias humanas y sociales. Se compone de estudios originales relacionados con la disciplina histórica así como su didáctica y difusión. Las diferentes secciones que componen la revista son: artículos de investigación, entrevistas a profesionales, recensiones de monografías de actualidad y crónicas de congresos o eventos científicos relevantes.

Todos los artículos publicados son objeto de un proceso de revisión a cargo de un mínimo de dos evaluadores, que se consideran expertos en el ámbito temático del artículo propuesto. Nuestro deseo es poder ofrecer unos contenidos rigurosos, de calidad y de interés.

EI CEPOAT (Centro de Estudios del Próximo Oriente y la Antigüedad Tardía de la Universidad de Murcia) es la institución encargada de la coordinación y gestión de la revista, desde donde anualmente se lanzará la convocatoria para aquellos que estén interesados en publicar sus trabajos, siempre relacionados con la Historia, Arqueología, Historia del Arte, Didáctica de las Ciencias Sociales, etc.

PANTA REI is a digital journal focused on History and other sciences related to it. Its main objective is the transmission of scientific knowledge by giving also an opportunity to young researchers who want to make their way in the study of human and social sciences. It is composed by original studies related to History, as well as its didactics and promotion. The different sections of this journal are: research articles, interviews to professionals, recensions on monographs about current issues and reports about congresses or relevant scientific events.

All the articles published are subject to a revision process carried out by a minimum of two reviewers who are considered to be experts in the field of the article proposed. Our wish is to offer rigorous contents with quality and being of interest to the reader.

CEPOAT (Centre of Studies of the Middle East and Late Antiquity of the University of Murcia) is the institution in charge of the coordination and management of this journal. This is the centre from where the call for papers will be launched for all the people interested in publishing their papers, always related to History, Archeology, Art History, Didactics of the Social Sciences, etc. 


\section{Normas de Publicación}

El autor se compromete a enviar trabajos originales, que no se encuentren publicados en otras revistas ni en otros idiomas. Así mismo, el mismo artículo no podrá ser presentado en otras revistas mientras dure el proceso de evaluación.

\section{Envío y presentación de originales}

Los artículos se enviarán exclusivamente a través del correo electrónico a la dirección pantarei@um.es. Los textos serán enviados en formato DOC y las imágenes en formato JPEG o TIFF, y con un tamaño mínimo de 2000 px. Éstas no aparecerán incorporadas en el texto, sino enviadas en archivo aparte y correctamente numeradas según su posición en el texto. Junto al trabajo, se rellenará y enviará un documento aparte en el que se especifiquen los datos del autor siguiendo el modelo disponible en la página Web de la revista.

Para la redacción de los trabajos se tendrá en cuenta el Manual de la American Psychological Association, en su sexta edición. La extensión máxima de los trabajos será de 30 páginas. La tipografía será Arial 11, con interlineado sencillo y sin espacio alguno entre párrafos. El texto deberá ir justificado a ambos márgenes y sin sangría en los primeros párrafos. Los márgenes serán de $2,50 \mathrm{~cm}$. En los casos en los que fuera necesario incorporar notas, éstas irán a pie de página, enumeradas consecutivamente, con tipografía Arial 10, interlineado sencillo y justificadas a ambos márgenes.

Una información más detallada se encuentra disponible en la página http://www.um.es/cepoat/ pantarei.

\section{Proceso de valoración y evaluación}

Una vez recibidos los trabajos, la Revista realizará una primera valoración. Si el trabajo enviado se ajusta a las normas de presentación propuestas, la temática es coincidente con la línea editorial de la revista y posee la calidad científica necesaria, será remitido al consejo asesor para una primera evaluación. Si no es así en este primer paso se puede rechazar directamente los documentos que incumplan claramente la línea editorial.

Será el Consejo Asesor quien indique a la revista la originalidad, relevancia, estructura, redacción, aparato bibliográfico, etc. del trabajo enviado y, para ello, se designará a dos revisores expertos externos que evaluarán cada uno de los trabajos, que pueden formar parte (o no) de este Consejo Asesor. La selección de los revisores se ajustará a la temática y características metodológicas del trabajo. El nombre y filiación de los autores serán eliminados del trabajo para su revisión, así como los revisores actuarán de manera anónima y confidencial.

Los revisores deberán rellenar un informe de evaluación que centrará su atención en aspectos tales como características formales, originalidad y novedad de los trabajos, relevancia de las propuestas y los resultados, calidad metodológica y validez científica.

Una vez terminado el proceso se decidirá la aceptación o no de los mismos y su publicación en el número que sea pertinente, así como las modificaciones susceptibles de ser realizadas para su final publicación. Dicha notificación se enviará únicamente por correo electrónico, en un plazo máximo de seis meses. 


\section{Publishing rules}

The author is committed to submit original papers not having been published in other reviews or in other languages. In this way, it is not allowed for the same paper to be presented in other reviews during the evaluation process.

\section{Submission and presentation of originals}

The articles will be exclusively submitted by email to pantarei@um.es. The texts will be submitted in DOC format and the images in JPEG or TIFF format, and with a minimum size of 2000 px. Images will not be integrated in the text but sent in another file and properly numbered according to their position in the text. Attached to the paper, a document will be filled out and sent where the author's data will be specified following the model available on the website.

The sixth edition of the Manual of the American Psychological Association will be taken into account for the writing of the papers. The length of the papers must not exceed 30 pages. Typography will be Arial 11 , with simple line spacing and no space between paragraphs. The text must be justified on both margins without indentation in the first paragraphs. Margins size will be $2.50 \mathrm{~cm}$. Where it could be necessary the incorporation of notes, they will be at the bottom of the page, consecutively numbered with typography Arial 10, simple line spacing and justified on both margins.

More detailed information is available on the website: http://www.um.es/cepoat/pantarei.

\section{Examination and assessment process}

The Journal will submit the papers to a first examination once received. If the paper follows the presentation guidelines, the subject agrees with the editorial line of this journal, and possess the scientific quality required, it will be sent to the advisory council for a first assessment. If not, the documents which clearly fail to complete the editorial line may be rejected straightaway in this first step.

The Advisory Council will indicate the originality, relevance, structure, writing, bibliography, etc. of the text to the journal; for this purpose, two outside experts will be designated to review the papers; these experts can be (or not) part of this Advisory Council. The selection of the experts will adjust to the subject and methodological characteristics of the paper. Name and affiliation of the author will be eliminated from the text for its review, in this way experts will act anonymously and confidentially.

The experts will fill out an assessment report which will focus on aspects such as formal characteristics, originality and novelty of the papers, relevance and results of the proposal, methodological quality and scientific validity.

Once the process is finished, the acceptance or not of the papers and its publication in the corresponding edition will be decided, as well as the modifications that may be done for its final publication. This notification will be sent by email within 6 months maximum. 

